\title{
Registro de un Monstruo Diprósopo de Tiburón Azul (Prionace glauca) en Chile
}

\author{
Record of a Diprosopus Monster of Blue Shark (Prionace glauca) from Chile \\ Valentina Hevia-Hormazábal; Víctor Pastén-Marambio \& Alonso Vega
}

HEVIA-HORMAZÁBAL, V.; PASTÉN-MARAMBIO, V. \& VEGA, A. Registro de un monstruo diprósopo de tiburón azul (Prionace glauca) en Chile. Int. J. Morphol., 29(2):509-513, 2011.

RESUMEN: Un espécimen de siameses de tiburón azul, Prionace glauca, fue recolectado durante actividades de pesca en el norte de Chile. Según una clasificación basada en el grado de duplicación y el sitio de fusión de los gemelos, el espécimen corresponde a un monstruo del tipo diprósopo tetroftalmo. El individuo tiene una duplicación craneofacial parcial con presencia de cuatro globos oculares e igual número de narinas. Las cavidades orales están interconectadas convergiendo en una única faringe. El tronco es simple; la columna presenta lordosis torácica y una torsión helicoidal en su porción caudal. Este es el primer reporte de siameses en condrictios chilenos y primer registro mundial de diprosopa en tiburones.

PALABRA CLAVE: Elasmobranquios, Gemelos siameses; Diprosopa.

\section{INTRODUCCIÓN}

Los siameses son gemelos parcialmente fusionados (Lattus et al., 2002), ocurren exclusivamente cuando comparten un mismo ambiente gestacional y se clasifican clínicamente como gemelos monocoriónico-monoamnióticos (Kaufman, 2004). Tienen una baja incidencia demográfica y pocas expectativas de supervivencia (Insunza et al., 2005); no obstante, el número de registros y descripciones respecto a éstos es alto (Lattus et al.; Kaufman). El cancerbero de la mitología griega y los monstruos circenses del siglo XVIII constituyen un testimonio de su presencia a lo largo de la historia (Moros, 2003).

Hasta la fecha, se han documentado casos de siameses en mamíferos (Lattus et al.; Vale-Echeto et al., 2004; Kompanje \& Hermans, 2008), aves (Ulshafer \& Clavert, 1979), reptiles (Matz, 2001), anfibios (Neff et al., 1990) y peces (Behnke \& Kloppel, 1975; Owusu-Frimpong \& Hargreaves, 2000; Al-Jufaily et al., 2005). En tiburones, los reportes de esta malformación son relativamente escasos, pero existen en Squalus acanthias Linnaeus (Bosinceano, 1934), S. blainvillei (Risso) (Lozano-Cabo, 1945), Rhizoprionodon acutus (Rüppell) (como Carcharias walbeehmi (Bleeker), Gopalan, 1971), Galeorhinus galeus Linnaeus (Delpiani et al., 2011) y Prionace glauca
(Linnaeus) (Goto et al., 1981), todos de monstruos bicéfalos (Palomero et al., 1998). Además, se han descrito siameses toracópagos en S. acanthias Linnaeus (como Acanthias vulgaris Risso, Ford, 1930). Otras anormalidades morfológicas reportadas en tiburones son, principalmente, albinismo y malformaciones esqueléticas (Mancini et al., 2006; Saïdi et al., 2006; Olatunji-Akioye et al., 2010).

El presente trabajo tiene como objetivo describir y clasificar un espécimen de siameses de $P$. glauca encontrados en aguas chilenas. En Chile, $P$. glauca se distribuye desde Arica a Puerto Montt (Pardo-Gandarillas et al., 2007). Es una especie vivípara, con un período gestacional de 9 a 12 meses (Nakano \& Seki, 2003), tras el cual cada hembra da a luz entre 4 y 135 crías por camada (Compagno, 1984).

\section{MATERIAL Y MÉTODO}

En verano de 1998, durante faenas de pesca realizadas en el norte de Chile se obtuvo por captura incidental (Lamilla, 2005), un ejemplar de siameses de $P$. glauca. El espécimen se fijó en formalina al $10 \%$ y se conservó en etanol 
HEVIA-HORMAZÁbAL, V.; PASTÉN-MARAMBiO, V. \& VEGA, A. Registro de un monstruo diprósopo de tiburón azul (Prionace glauca) en Chile. Int. J. Morphol., 29(2):509-513, 2011.

Tabla I. Caracterización morfométrica (en mm) del monstruo diprósopo tetroftalmo capturado en aguas chilenas y de un juvenil normal de P. glauca.

\begin{tabular}{|c|c|c|c|c|c|}
\hline & \multicolumn{2}{|c|}{ Diprósopo } & \multicolumn{3}{|c|}{ Normal } \\
\hline & Izquierda & Derecha & Izquierda & & Derech \\
\hline Largo total & 152 & 160 & & 431 & \\
\hline Distancia entre hocicos & & & & - & \\
\hline Desde el hocico hasta: & & & & - & \\
\hline narina exterior & 15 & 14 & 24 & & 24 \\
\hline narina interior & 12 & 14 & & - & \\
\hline ojo interior & 22 & 24 & 41 & & 41 \\
\hline ojo exterior & 30 & 31 & & - & \\
\hline Boca & 34 & 33 & & 46 & \\
\hline $1^{\mathrm{a}}$ hendidura branquial & 64 & 65 & 89 & & 91 \\
\hline $3^{\mathrm{a}}$ hendidura branquial & 74 & 74 & 93 & & 98 \\
\hline $5^{\text {a }}$ hendidura branquial & 79 & 80 & 99 & & 107 \\
\hline origen aleta pectoral & 76 & 79 & 102 & & 104 \\
\hline origen aleta pélvica & 122 & 123 & & 201 & \\
\hline origen $1^{\mathrm{a}}$ dorsal & 118 & 112 & & 172 & \\
\hline origen $2^{\mathrm{a}}$ dorsal & 139 & 142 & & 266 & \\
\hline origen aleta anal & 122 & 131 & & 251 & \\
\hline Distancia inter-narial interior: & 12 & 11 & & 19 & \\
\hline \multicolumn{6}{|l|}{ Boca: } \\
\hline Ancho & 19 & 17 & & 27 & \\
\hline Alto & 11 & 12 & & 20 & \\
\hline \multicolumn{6}{|l|}{ Longitud de hendidura branquial : } \\
\hline $1^{\circ}$ & 7 & 4 & 13 & & 13 \\
\hline $2^{\circ}$ & 8 & 5 & 12 & & 12 \\
\hline $3^{\circ}$ & 7 & 6 & 12 & & 12 \\
\hline $4^{\mathrm{o}}$ & 6 & 5 & 10 & & 10 \\
\hline $5^{\circ}$ & 7 & 4 & 11 & & 11 \\
\hline Ojo (diámetro horizontal): & & & 13 & & 13 \\
\hline Interior & 13 & 12 & & - & \\
\hline Exterior & 11 & 11 & & - & \\
\hline \multicolumn{6}{|l|}{$1^{\text {a }}$ Aleta dorsal: } \\
\hline longitud margen anterior & & & & 35 & \\
\hline longitud de la base & & & & 28 & \\
\hline longitud margen posterior & & & & 14 & \\
\hline Altura & & & & 19 & \\
\hline \multicolumn{6}{|l|}{$2^{\mathrm{a}}$ Aleta dorsal } \\
\hline longitud margen anterior & & & & 17 & \\
\hline longitud de la base & & & & 16 & \\
\hline longitud margen posterior & & & & 15 & \\
\hline Altura & & & & 8 & \\
\hline \multicolumn{6}{|l|}{ Aleta anal: } \\
\hline longitud general & & & & 22 & \\
\hline longitud de la base & & & & 17 & \\
\hline longitud margen posterior & & & & 11 & \\
\hline Altura & & & & 8 & \\
\hline \multicolumn{6}{|l|}{ Aleta pectoral: } \\
\hline longitud de la base & 9 & 11 & 12 & & 12 \\
\hline longitud margen anterior & 48 & 48 & 70 & & 70 \\
\hline longitud del borde libre & 12 & 12 & 19 & & 19 \\
\hline longitud margen posterior & 26 & 27 & 50 & & 50 \\
\hline \multicolumn{6}{|l|}{ Aleta pélvica: } \\
\hline longitud de la base & - & 11 & 22 & & 22 \\
\hline longitud margen anterior & 7 & 24 & 24 & & 24 \\
\hline longitud margen posterior & - & 14 & 14 & & 14 \\
\hline Ancho tronco en $4^{\mathrm{a}}$ hendidura braquial: & & & & 41 & \\
\hline
\end{tabular}


al 90\%; posteriormente, se ingresó a la colección ictiológica del Campus Guayacán de la Universidad Católica del Norte (UCN), donde se identificó mediante claves de Chirichigno \& Vélez (1998) y de Sáez \& Pequeño (2010). Para el análisis descriptivo del ejemplar, se efectuaron pruebas radiológicas, un examen morfológico externo y una caracterización morfométrica según Compagno. Paralelamente, se compararon las medidas corporales del espécimen de siameses con las de un individuo normal de la misma especie, sexo y edad. La clasificación de los siameses se realizó siguiendo los criterios de Fisher (1866) y de Palomero et al., basados en la simetría, duplicidad y área de fusión gemelar.

\section{RESULTADOS Y DISCUSIÓN}

El ejemplar analizado corresponde a siameses nonatos hembras, longitud total: $160 \mathrm{~mm}$, cuyas proporciones morfométricas difieren completamente de las observadas en el individuo normal (Tabla I). Su caracterización morfológica muestra duplicidad craneofacial parcial, con cabezas lateralmente unidas, cuatro globos oculares e igual número de narinas (Fig. 1a). Tanto los condrocráneos como las mandíbulas superiores ofrecen un aspecto externo normal, mientras que las mandíbulas inferiores exhiben una curvatura anormal para la especie (Fig. 1). Los ojos mediales comparten una misma órbita y están separados por un estrecho tabique conjuntivo (Fig. 1a); las cavidades orales se fusionan posteriormente y convergen en un conducto faríngeo común (Fig. 1b). El tronco es simple, con cordón umbilical persistente (Fig. 1a), dotación completa de hendiduras branquiales (Tabla I, Fig. 1b) y número normal de aletas (Tabla I, Fig. 1). Las aletas pectorales son asimétricas y la anal, rudimentaria (Fig. 1a). El esqueleto axial presenta lordosis torácica (Fig. 1b) y torsión helicoidal dextrógira a través de su porción caudal (Fig. 1).

Según la clasificación general de Palomero et al., el ejemplar descrito en este estudio se adscribe a la categoría monstruos, gemelos que comparten la mayoría de sus órganos internos y cuya estrecha relación anatómica hace inviable su separación quirúrgica. Además, la presencia de un tronco unitario y la duplicidad parcial de sus elementos craneofaciales permiten subclasificarlo como diprosopus tetroftalmus (Fisher).

La existencia de cuatro tractos ópticos sugiere el desarrollo de dos canales espinales distintos, los cuales confluyen a nivel cervical (Fig. 2a). La coalescencia craneocervical, específica de los siameses diprósopos, define al espécimen estudiado y lo diferencia de bicéfalos descritos para P. glauca (Fig. 2b) y otras especies de tiburones (Bosinceano; Lozano-Cabo; Gopalan; Goto et al.; Delpiani et al.).
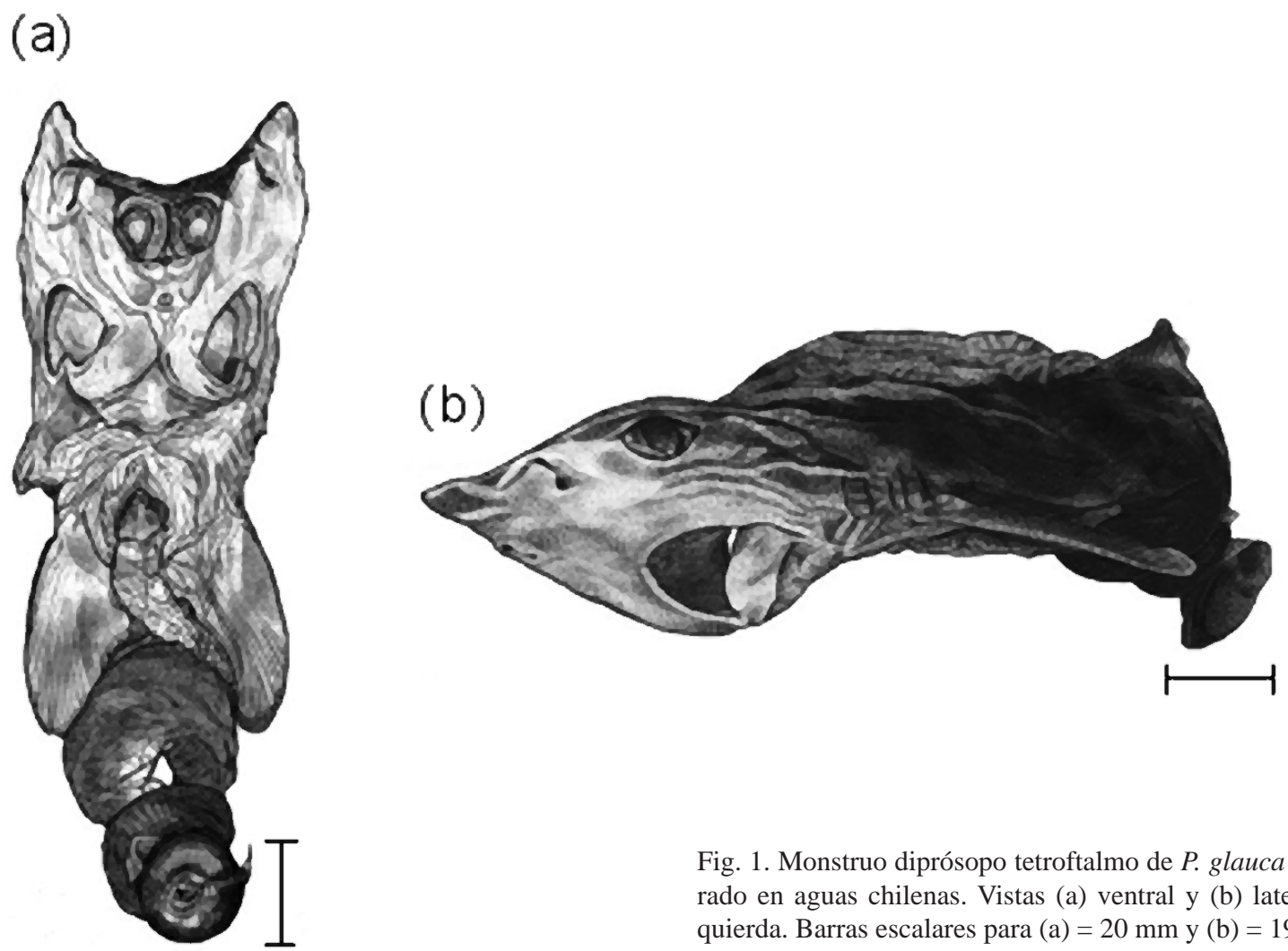

Fig. 1. Monstruo diprósopo tetroftalmo de P. glauca capturado en aguas chilenas. Vistas (a) ventral y (b) lateral izquierda. Barras escalares para $(\mathrm{a})=20 \mathrm{~mm}$ y $(\mathrm{b})=19 \mathrm{~mm}$. 

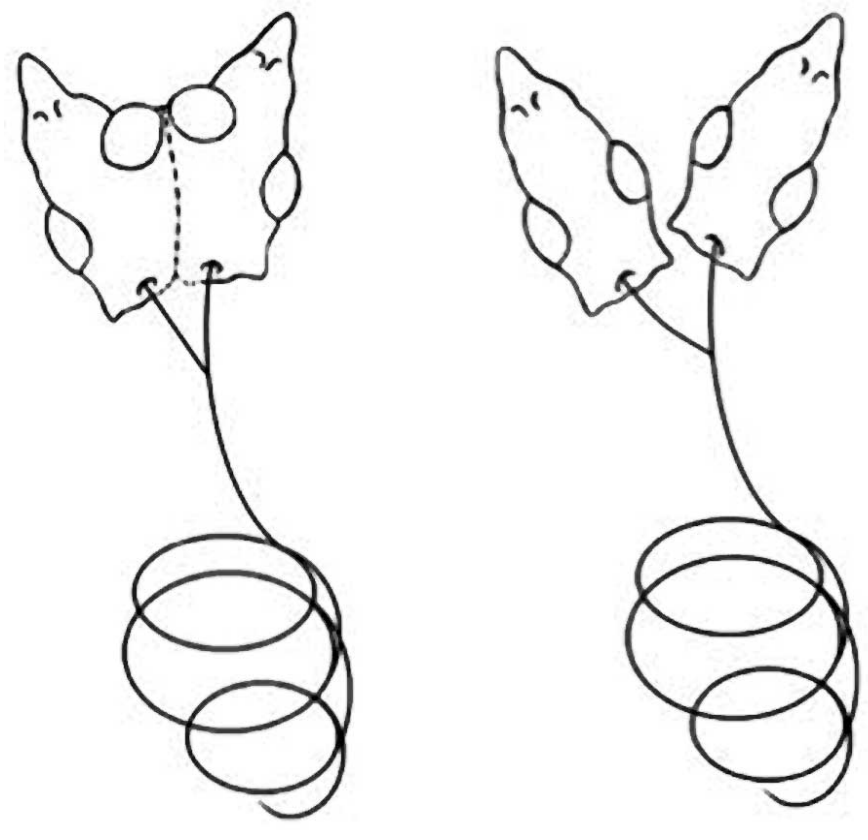

Fig. 2. Esquema comparativo del monstruo diprósopo tetroftalmo (a) descrito en este estudio y de un monstruo bicéfalo (b) de $P$. glauca mostrado por Goto et al. (1981).
De acuerdo con los resultados obtenidos, el presente trabajo constituye el primer reporte de siameses para la fauna de condrictios chilenos, y el primer registro mundial de diprosopía en tiburones.

Con respecto a la génesis de los siameses, se han propuesto dos mecanismos alternativos: división incompleta del disco embrionario y fusión secundaria de embriones adyacentes (Kaufman; Molina et al., 2008). En mamíferos, anfibios y peces, agentes como hipoxia, hipertermia, centrifugación y químicos de acción teratogénica han permitido la inducción experimental de gemelos fusionados (Neff et al.; Owusu-Frimpong \& Hargreaves; Kaufman). En tiburones, su etiología estaría asociada a factores como malnutrición, parasitosis, anomalías genéticas (Delpiani et al.), elevada producción de embriones y degradación ambiental (Mancini et al.).

AGRADECIMIENTOS. Agradecemos a M. Godoy por recolectar y depositar el espécimen en el museo UCN, la colaboración de E. Acuña, S. Akaboshi, N. Piaget, J. Muñoz, G. Montecinos, E. von Brand y G. Díaz; y el apoyo de FONDECYT a través del Proyecto $N^{\circ} 1070417$.

HEVIA-HORMAZÁBAL, V.; PASTÉN-MARAMBIO, V. \& VEGA, A. Record of a diprosopus monster of blue shark (Prionace glauca) from Chile. Int. J. Morphol., 29(2):509-513, 2011.

SUMMARY: A conjoined twins specimen of blue shark Prionace glauca was collected during fishing activities in Northern Chile. According to a classification based on duplication degree and fusion site of the twins, it represents a monster of diprosopus tetrophthalmus type. The specimen has a partial craniofacial duplication with presence of four eyeballs and an equal number of nostrils. Oral cavities are interconnected, converging in a single pharynx. The trunk is simple; the vertebral column presents thoracic lordosis and helical torsion in its caudal portion. This is the first Chilean record of conjoined twins to chondrichthyan and first world record of diprosopia in sharks.

KEY WORDS: Elasmobranchs; Conjoined twins; Diprosopia.

\section{REFERENCIAS BIBLIOGRÁFICAS}

Al-Jufaily, S. M.; Jawad, L. A. \& Al-Azi, A. N. Wild Siamesetwins in black tip sea catfish, Arius dussumieri (Valencienes, 1840) from Gulf of Oman. An. Biol., 27:2235,2005 .

Behnke, R. J. \& Kloppel, T. M. An expression of unequal twinning in an adult rainbow trout. Copeia, 4:775-7, 1975.

Bosinceano, A. Sur un cas de monstre double incomplet chez Squalus acanthias (On a case of incomplete double monster in Squalus acanthias). Ann. Univ. Sci. Jassy, 19:339-44, 1934.

Chirichigno, N. \& Vélez, J. Clave para identificar los peces marinos del Perú. Callao, Instituto del Mar del Perú, 1998.
Compagno, L. J. V. FAO Species Catalogue. Vol. 4. Sharks of the world: An annotated and illustrated catalogue of sharks' species known to date. Part 2. Carcharhiniformes. FAO Fish Synop., 125(4):251-655, 1984.

Delpiani, S. M.; Deli Antoni, M. Y.; Barbini, S. A. \& Figueroa D. E. First record of a dicephalic specimen of tope Galeorhinus galeus (Elasmobranchii: Triakidae). J. Fish Biol., 78(3):941-4, 2011.

Fisher, G. J. Diploteratology. Transactions of the Medical Society of the State of New York, 1866, pp.227-31.

Ford, E. Some abnormal fishes received at the Plymouth Laboratory. J. Mar. Biol. Assoc. U. K., 17(1):53-64, 1930. 
Gopalan, U. K. On two abnormal sharks from Gujarat. J. Bomb. Nat. Hist. Soc., 68:465-6, 1971.

Goto, M.; Taniuchi, T.; Kuga, N. \& Iwata, M. Four dicephalous specimens of the blue shark, Prionace glauca, from Japan. Japan. J. Ichthyol., 28(2):157-65, 1981.

Insunza, A.; Carrillo, J.; Yamamoto, M.; Valentini, P. \& Ferrand, P. Aborto retenido con fetos toracópagos. Rev. Chil. Obstet. Ginecol., 70(1):21-3, 2005.

Kaufman, M. The embryology of conjoined twins. Childs Nerv. Syst., 20(8-9):508-25, 2004.

Kompanje, E. J. O. \& Hermans, J. J. Cephalopagus conjoined twins in a Leopard Cat (Prionailurus bengalensis). J. Wildlife Dis., 44(1):177-80, 2008.

Lamilla, J. Bycath: Tiburones en peligro. OCEANA, 11:1-14, 2005.

Lattus, J.; Almuna, R.; Paredes, A.; Junemann, K.; Guerra, F.; Pizarro, O.; Zúñiga, M.; Martic A. \& Missarelli, C. Siameses o gemelos unidos toraco-onfalópagos y revisión de bibliografía nacional e internacional. Rev. Chil. Obstet. Ginecol., 67(5):392-401, 2002.

Lozano Cabo, D. Nota sobre un caso de bicefalismo en el Squalus blainvillei (Note on a case of bicephalism in Squalus blainvillei ). Bol. R. Soc. Esp. Hist. Nat. (Sec. Biol.), 43:147-8, 1945.

Matz, G. Tératologie. En: Bauchot, R. (Ed). Serpents. Paris, Artemis, 2001. pp.106-7.

Mancini, P. L.; Casas, A. L. \& Amorim, A. F. Morphological abnormalities in a blue shark Prionace glauca (Chondrichthyes: Carcharhinidae) foetus from Southern Brazil. J. Fish Biol., 69:1881-4, 2006.

Moros, M. Seres extraordinarios. Anomalías, deformidades y rarezas humanas. $1^{\text {a }}$ ed. Madrid, Edaf, 2003.

Molina, S.; Duque, J.; Motta, A.; Alvarado, C. \& Torres, C. Duplicación facial Diprosopus: Reporte de un caso y revisión de la literatura. Latin American Journal of Dysmorphology, 1:10-4, 2008.

Nakano, H. \& Seki, M. Synopsis of biological data on the blue shark, Prionace glauca Linnaeus. Bull. Fish. Res. Agency, 6:18-55, 2003.

Neff, A. W.; Wakahara, M. \& Malacinski, G. M. Bifurcation of the amphibian embryo's axis: analysis of variation in response to egg centrifugation. Int. J. Dev. Biol., 34:391-8, 1990.
Olatunji-Akioye, A. O.; Adeyemo, O. K. \& Akomolafe, O. T. Photographic and radiographic study of osteological abnormalities of the head of adult African catfish (Clarias gariepinus). Int. J. Morph., 28(3):719-22, 2010.

Owusu-Frimpong, M. \& Hargreaves, J. A. Incidence of conjoined twins in tilapia after thermal shock induction of polyploidy. Aquacul. Res., 31(5):421-6, 2000.

Palomero, G.; Vásquez, M.; Vega, J.; Naves, F. \& Rodríguez, C. Lecciones de embriología. Asturias, Servicio de Publicaciones de la Universidad de Oviedo, 1998.

Pardo-Gandarillas, M.; Duarte, F.; Chong, J. \& Ibáñez, C. Dieta de tiburones juveniles Prionace glauca (Linnaeus, 1758) (Carcharhiniformes: Carcharhinidae) en la zona litoral centro-sur de Chile. Rev. Biol. Mar. Oceanogr., 42(3):3659, 2007.

Sáez, S. \& Pequeño, G. Claves para el reconocimiento taxonómico dentario en taxa del Superorden Squalomorphi de Chile (Chondrichthyes: Elasmobranchii) Lat. Am. J. Aquat. Res., 38(3):474-84, 2010.

Saïdi, B.; Bradaï, M. N.; Marouani, S.; Guélorget, O. \& Capapé, C,. Atypical characteristics of an albino embryo of Carcharhinus plumbeus (Chondrichthyes: Carcharhinidae) from the Gulf of Gabès (Southern Tunisia, central Mediterranean). Acta Adriatica, 47(2):167-74, 2006.

Ulshafer, R. J. \& Clavert, A. The use of avian double monsters in studies on induction of the nervous system. J. Embr. Exp. Morph., 53:237-43, 1979.

Vale-Echeto, O.; Alvarado, M.; Vale-Oviedo, O.; Árraga C.; Camacho, J. \& Fernández, E. Duplicidad parcial cefálicafacial (diprosopia) en bovinos: estudio clínico patológico de un caso. Rev. Cient., 14(4):338-43, 2004.

Dirección para correspondencia:

M. Cs. J. M. Alonso Vega Reyes

Universidad Católica del Norte

Coquimbo

CHILE

Email: avega@ucn.cl

Recibido : 21-03-2011

Aceptado: 11-04-2011 